\title{
Nivolumab and ipilimumab combination promises new standard of care for advanced $\mathrm{RCC}$
}

$I^{\mathrm{s}}$ n April 2018, the US Food and Drug Administration expanded the approval of the combination of nivolumab and ipilimumab into a new indication, following a previous approval in patients with metastatic melanoma. The double immune checkpoint inhibitor combination was approved on the basis of the phase 3 CheckMate-214 study for the treatment of patients with intermediate- or poorrisk, previously untreated advanced renal cell carcinoma (RCC). ${ }^{1}$

Nivolumab monotherapy is already approved in the second-line setting for the treatment of advanced RCC, and the demonstration of significantly improved overall survival (OS) in this study suggests that the combination should supplant sunitinib in the front-line setting in the treatment of this type of cancer.

A total of 1,096 patients at 175 sites in 28 countries were randomized $1: 1$ to receive nivolumab $(3 \mathrm{mg} / \mathrm{kg})$ and ipilimumab $(1 \mathrm{mg} / \mathrm{kg})$ intravenously every 3 weeks for 4 doses in an induction phase, followed by nivolumab monotherapy ( $3 \mathrm{mg} / \mathrm{kg}$ ) every 2 weeks in a maintenance phase or sunitinib $(50 \mathrm{mg}$ ) orally daily for 4 weeks of each 6-week cycle.

Eligible patients were 18 years or older, had previously untreated advanced RCC with a clear-cell component, had measurable disease according to Response Evaluation Criteria in Solid Tumors (version 1.1), and had a Karnofsky performance status of at least 70 (on a scale from 0 to 100 , with lower scores indicating greater disability). Patients with central nervous system metastases or autoimmune disease who were being treated with glucocorticoids and immunosuppressants were excluded from the study.

Around three-quarters of patients with advanced RCC have intermediate- or poor-risk disease and experience worse outcomes than patients with favorable-risk disease. Patients in CheckMate-214 were stratified according to International Metastatic Renal Cell Carcinoma Database Consortium risk score as favorable (score of 0 ), intermediate (score of 1 or 2 ) or poor risk (score of 3-6), according to the number of risk factors present.

Risk factors included a Karnofsky performance score of 70, time from initial diagnosis to randomization of $<1$ year, a hemoglobin level below the lower limit of normal, a corrected serum calcium concentration of $>10 \mathrm{mg} / \mathrm{dL}$, or

\section{What's new, what's important}

This approval of the nivolumab-ipilimumab combination for patients with advanced RCC heralds a new standard of care RCC that will likely sideline sunitinib as a first-line therapy given the significant improvements in OS with the double-immunotherapy combination.

The approval was informed by findings from the phase 3 CheckMate-214 study in which patients received either the nivolumab-ipilimumab combination or sunitinib alone. Patients were stratified by risk score (favorable, intermediate, poor risk) and by geographic region. The endpoints were ORR, PFS, and OS in intermediate- and poor-risk patients. Over a median follow-up of 25.2 months, there was a significant improvement in OS and ORR in the study group patients (mPFS not reached; ORR, 41.6\%), compared with the controls (OS, 25.9 months; ORR, 26.5\%), with $\mathrm{P}<.001$ for both. The combination was favored across subgroups.

The most common AEs with the immunotherapy combination included fatigue, rash, diarrhea, musculoskeletal pain, pruritus, nausea, and others. The combination was associated with fewer grade $3 / 4$ AEs ( $63 \%$ vs $46 \%$ for sunitinib), but a higher rate of AE-related treatment discontinuations ( $31 \%$ vs $21 \%)$. The study group had 8 treatment-related deaths; the control group, 4 .

Warnings include mostly immune-mediated AEs, and risk of infusion reactions and for embryofetal toxicity. Patients should be monitored for hyperglycemia and for changes in liver, thyroid, renal, and neurologic function. New-onset moderate to severe neurologic signs or symptoms warrant treatment being withheld, and immune-mediated encephalitis should lead to treatment discontinuation. Patients should be advised of the potential for fetal harm and the need for effective contraception during and after treatment.

$$
\text { - Jame Abraham, MD, FACP (abrahaj5@ccf.org) }
$$

an absolute neutrophil count or platelet count above the upper limit of normal. Patients were also stratified according to geographic region (United States versus Canada and Europe versus the rest of the world).

The coprimary endpoints were objective response rate (ORR), progression-free survival (PFS), and OS in a subset

Report prepared by Jane de Lartigue, PhD. JCSO 2018;16(5):e182-e184. (2018 Frontline Medical Communications. doi: https://doi. org/10.12788/jcso.0421 


\section{Mechanism of action: immune checkpoint inhibitors}

\section{Dual blockade of immune check-} points. T cells are central effectors of the adaptive immune response and have also been shown to be activated in response to tumor-cell antigens as part of the antitumor immune response, with many tumor types demonstrating high levels of infiltrating $T$ cells in the tumor microenvironment.

To mount an effective immune response, T cells must receive 2 signals, 1 from the T-cell receptor, which is activated by antigen presented by specialized immune cells, and a secondary signal that essentially decides whether the $T$ cell is turned on or off in response to the particular antigen.

The secondary signal is often referred to as an immune checkpoint, can be either stimulatory (the on switch) or inhibitory (the off switch), and helps to ensure that T-cell-mediated immunity is able to eliminate a threat without causing any collateral damage to healthy tissue. It can also be exploited by tumor cells to help them evade the anti-tumor immune response by switching off infiltrating $T$ cells.

Programmed cell death-1 receptor (PD-1) and cytotoxic T-lymphocyte antigen-4 (CTLA-4) are 2 of the main inhibitory signals and their ligands are often expressed by tumors. Immune checkpoint inhibitors (drugs targeting these receptors and their ligands) have been successfully used as anticancer therapeutics and are being approved in an expanding range of tumor types.

Nivolumab, which targets PD-1, in particular has proved highly effective as monotherapy. However, a significant number of patients don't respond to nivolumab or develop resistance. Ipilimumab targets CTLA-4 and has been approved for the treatment of metastatic melanoma. Due to their distinct mechanisms of action on different T-cell inhibitory pathways, a combination of nivolumab and ipilimumab has demonstrated synergistic antitumor activity in preclinical models and the combination has already been approved for the treatment of metastatic melanoma. of 847 intermediate- and poor-risk patients. Over a median follow-up of 25.2 months, there was a statistically significant improvement in OS and ORR in patients treated with nivolumab and ipilimumab (mPFS not reached; ORR, 41.6\%), compared with sunitinib (OS, 25.9 months; ORR, $26.5 \%$ ), with $P<.001$ for both. The immunotherapy combination was favored across subgroups.

The most common adverse events (AEs) in patients treated with nivolumab and ipilimumab included fatigue, rash, diarrhea, musculoskeletal pain, pruritus, nausea, cough, pyrexia, arthralgia, and decreased appetite. The combination was associated with fewer grade 3/4 AEs (63\% vs $46 \%$ for sunitinib), but a higher rate of treatment discontinuations because of AEs (31\% vs 21\%, respectively). There were 8 deaths in the combination arm, and 4 in the sunitinib arm that were reported to be treatment related. ${ }^{2}$
The warnings and precautions related to nivolumabipilimumab combination therapy outlined in the prescribing information include mostly immune-mediated AEs, such as immune-mediated pneumonitis, colitis, hepatitis, endocrinopathies, nephritis and renal dysfunction, skin adverse reactions, and encephalitis. There are also warnings relating to the risk of infusion reactions and the potential for embryofetal toxicity.

Patients should be monitored for hyperglycemia and for changes in liver, thyroid, renal, and neurologic function. Treatment with nivolumab and ipilimumab should be withheld for moderate and permanently discontinued for severe or life-threatening immune-mediated pneumonitis, colitis, and hepatitis, as well as transaminase or total bilirubin elevation. It should also be withheld for moderate or severe hypophysitis and serum creatinine 


\section{Community Translations}

elevation, moderate adrenal insufficiency and severe hyperglycemia, and permanently discontinued for lifethreatening hypophysitis and serum creatinine elevation, severe or life-threatening adrenal insufficiency, and lifethreatening hyperglycemia.

New-onset moderate to severe neurologic signs or symptoms warrant treatment being withheld, and immune-mediated encephalitis should lead to treatment

\section{References}

1. US Food and Drug Administration website. FDA approves nivolumab plus ipilimumab combination for intermediate or poorrisk advanced renal cell carcinoma. https://www.fda.gov/drugs/informationondrugs/approveddrugs/ucm604685.htm. Last updated April 16, 2018. Accessed July 25, 2018.

2. Motzer RJ, Tannir NM, McDermott O, et al. Nivolumab plus ipilimumab versus sunitinib in advanced renal-cell carcinoma. $\mathrm{N}$ Engl J Med. 2018;378:1277-1290. discontinuation. For mild or moderate infusion reactions, the infusion rate can be slowed or interrupted, and infusions should be discontinued in the event of severe or lifethreatening infusion reactions. Patients should be advised of the potential for fetal harm and the need for effective contraception during and after treatment. Ipilimumab and nivolumab are marketed as Yervoy and Opdivo, respectively, by Bristol-Myers Squibb., ${ }^{3,4}$

3. Opdivo (nivolumab) injection, for intravenous use. Prescribing information. Bristol-Myers Squibb. https://www.accessdata.fda.gov/ drugsatfda_docs/label/2018/125554s0581bl.pdf. Revised April 2018 Accessed September 16, 2018.

4. Yervoy (ipilimumab) injection, for intravenous use. Prescribing information. Bristol-Myers Squibb. July 2018. https://packageinserts. bms.com/pi/pi_yervoy.pdf. Accessed September, 2018. 